\title{
A GENERALIZATION OF SHELAH'S OMITTING TYPES THEOREM
}

\author{
By
}

Kota TAKeUCHI

\begin{abstract}
This note gives generalizations of Shelah's omitting types theorem and Lopez-Escobar's Theorem.
\end{abstract}

\section{Introduction}

The omitting types theorem states that for a given countable set $S$ of nonisolated types in $T$, there is a model of $T$ omitting all the members of $S$, where $T$ is a theory of a countable language. If $L$ is uncountable, it is easy to construct an $L$-theory, that is, a counter example to the omitting types theorem. So, we are always interested in a theory with a countable language. There are many generalizations of the theorem. Among these, Shelah's omitting types theorem is of special interest.

TheOREM (Shelah). Let $T$ be a theory of a countable language L. Let $R$ be a set of nonisolated complete types such that $|R|<2^{\omega}$. Then there is a model $M \models T$ omitting all the members of $R$.

If we assume Martin's Axiom, we can omit $<2^{\omega}$ nonisolated types. Newelski studied the maximum cardinal $\kappa$ such that we can omit $<\kappa$ nonisolated types. It is known that there is a model of $\mathrm{ZFC}+\neg \mathrm{CH}$ such that $\kappa=\omega_{1}$ (see [4]). So, we cannot omit the assumption of the completeness of types in Shelah's omitting types theorem.

One of the main theorems in this paper is the following; it simultaneously generalizes the usual omitting types theorem and Shelah's omitting types theorem, and is proved in section 3 .

AMS 2000 subject classification: 03C68, 03C95.

Keywords and phrases: Shelah's omitting types theorem, nonelementary class, uncountably many types, the omitting types theorem.

Received February 1, 2011.

Revised May 23, 2011. 
THEOREM. Let $T$ be a theory formulated in a countable language $L$ and $L_{0}$ a sublanguage of $L$. Let $R$ be a set of nonisolated complete $L_{0}$-types such that $|R|<2^{\omega}$. Let $S$ be a countable set of nonisolated L-types. Then there is a model $M \models T$ omitting all the members of $R \cup S$.

In section 4, we apply the above theorem to another version of the omitting types theorem, the Lopes-Escobar theorem [3]. The Lopes-Escobar theorem is as follows.

THEOREM (Lopez-Escobar). Let $T$ be a theory formulated in a countable language $L$ having a binary relation $<$. Let $S$ be a countable set of L-types. Suppose that for any $\alpha<\omega_{1}$, there is a model $M_{\alpha}$ of $T$ omitting $S$ and with the order type $\alpha$. Then there is a model $N \models T$ omitting $S$ and with the order type $\mathbf{Q}$.

This theorem has already been generalized for uncountably many complete types by Tsuboi [8]. We generalize the theorem to our situation.

THEOREM. Let $T$ be a theory formulated in a countable language $L$ and $L_{0} a$ sublanguage of $L$, which have a binary relation $<$. Let $R$ be a set of nonisolated complete $L_{0}$-types such that $|R|<2^{\omega}$. Let $S$ be a countable set of L-types. Suppose that for any $\alpha<\omega_{1}$, there is a model $M_{\alpha}$ of $T$ omitting all the members of $R \cup S$ and with the order type $\alpha$. Then there is a model $N \models T$ omitting all the members of $R \cup S$ and with the order type $\mathbf{Q}$.

The omitting types theorem is also studied in nonclassical logics, $L_{\omega_{1}, \omega}$ [2], $L(Q)$ [5], etc. Our generalization of the omitting types theorem implies that Shelah's omitting types theorem holds in $P C_{\delta}$-classes. Precise definitions are given in section 5 .

\section{Preliminaries and Notations}

Throughout, $L$ is a countable language and $T$ is a countable first-order theory formulated in $L$. ( $T$ may be incomplete.) We always work under $T$. $L$-formulas are denoted by $\varphi, \psi, \theta, \chi, \ldots$ We fix a sublanguage $L_{0} \subset L$. $L_{0}$-formulas are denoted by $\xi, \ldots$ We assume that $\varphi, \psi, \ldots, \xi, \ldots$ are satisfiable unless otherwise noted. Types are (possibly inocomplete) $L$-types over the empty set. We say a type $p(\bar{x})$ is a complete $L_{0}$-type if $p$ consists of only $L_{0}$-formulas, and if for every $\xi(\bar{x}) \in L_{0}, \xi$ or $\neg \xi$ is in $p$. 
Example 1. Let $L=\left\{R_{i}(x) \mid i<\omega\right\}$. Consider an $L$-theory $T$, where for every finite subset $F, G \subset \omega$ with $F \cap G=\varnothing$, there is an element $x$ satisfying $\bigwedge_{i \in F} R_{i}(x) \wedge \bigwedge_{j \in G} \neg R_{j}(x)$. Then $T$ is complete and not small. Moreover, there is no isolated complete type. Let $R$ be a set of complete types with $|R|<2^{\omega}$. Shelah's omitting types theorem says that there is a model $M \models T$ omitting all the members of $R$. Take an infinite-coinfinite subset $S \subset \omega$. Set $\Sigma(x)=$ $\left\{R_{i}(x) \mid i \in S\right\}$. Then

1. $\Sigma$ is a nonprincipal type,

2. $\Sigma$ has continuum many extensions to nonprincipal complete types.

So, it is not clear that there is a model $N \models T$ omitting all the members of $R \cup\{\Sigma\}$.

\section{A Proof of the Theorem}

The main idea of the proof is simple; construct continuum many models such that each type in $R \cup S$ is omitted by almost models. Then, there must be a model that omits all the members of $R \cup S$ because $|R \cup S|<2^{\omega}$. To prove the theorem, we make the following definitions.

DeFINITION 2. Let $L_{0} \subset L$ and $\varphi_{i}(\bar{x}) \in L$.

1. We say that two $L$-formulas $\varphi_{0}(\bar{x})$ and $\varphi_{1}(\bar{x})$ are $L_{0}$-separable in $\bar{x}^{\prime} \subset \bar{x}$ if there are $L_{0}$-formulas $\xi_{0}\left(\bar{x}^{\prime}\right)$ and $\xi_{1}\left(\bar{x}^{\prime}\right)$ such that $T \models \varphi_{k}(\bar{x}) \rightarrow \xi_{k}\left(\bar{x}^{\prime}\right)$ $(k=0,1)$, and $\xi_{0}$ and $\xi_{1}$ are incompatible in $T$.

2. We say $\varphi_{0}(\bar{x})$ and $\varphi_{1}(\bar{x})$ are essentially $L_{0}$-separable in $\bar{x}^{\prime} \subset \bar{x}$ if there are $L$-formulas $\varphi_{k}^{\prime}(\bar{x})(k=0,1)$ with $T \models \varphi_{k}^{\prime}(\bar{x}) \rightarrow \varphi_{k}(\bar{x})(k=0,1)$ such that $\varphi_{0}^{\prime}$ and $\varphi_{1}^{\prime}$ are $L_{0}$-separable in $\bar{x}^{\prime}$.

3. Let $\Phi=\varphi_{0}(\bar{x}), \ldots, \varphi_{n}(\bar{x})$ be a sequnece of $L$-formulas. We say that $\Phi$ is maximally $L_{0}$-separated if for each $i \neq j$ and each subsequence $\bar{x}^{\prime} \subset \bar{x}$, whenever $\varphi_{i}^{\prime}(\bar{x})$ and $\varphi_{j}^{\prime}(\bar{x})$ are essentially $L_{0}$-separable in $\bar{x}^{\prime}$ then they are $L_{0}$-separable in $\bar{x}^{\prime}$.

A maximally $L_{0}$-separated sequence $\Phi^{\prime}=\varphi_{0}^{\prime}(\bar{x}), \ldots, \varphi_{n}^{\prime}(\bar{x})$ will be called a maximal $L_{0}$-separation of $\Phi$ if $T \models \varphi_{i}^{\prime}(\bar{x}) \rightarrow \varphi_{i}(\bar{x})(i=0, \ldots, n)$.

Lemma 3. Let $\Phi=\varphi_{0}(\bar{x}), \ldots, \varphi_{n}(\bar{x})$ be L-formulas. Then there are L-formulas $\varphi_{i}^{\prime}(\bar{x})(i \leq n)$ such that $\Phi^{\prime}=\varphi_{0}^{\prime}(\bar{x}), \ldots, \varphi_{n}^{\prime}(\bar{x})$ is a maximal $L_{0}$-separation of $\Phi$.

Proof. Let $\bar{y} \subset \bar{x}$ and suppose that $\varphi_{i}(\bar{y})$ and $\varphi_{j}(\bar{y})$ are essentially $L_{0}$ separable in $\bar{y}$. Choose an $L$-formula $\varphi_{i}^{\prime}(\bar{x})$ and an $L$-formula $\varphi_{j}^{\prime}(\bar{x})$ witnessing the 
essential $L_{0}$-separability. Then we replace $\varphi_{i}(\bar{x})$ and $\varphi_{j}(\bar{x})$ by $\varphi_{i}^{\prime}(\bar{x})$ and $\varphi_{j}^{\prime}(\bar{x})$, respectively. We repeat this process (finitely many times) and finally we get a desired maximal $L_{0}$-separation.

Definition 4. Let $\psi\left(x_{1}, \ldots, x_{n}\right)$ be an $L$-formula and $s(\bar{y})$ an $L$-type. We say $\psi\left(x_{1}, \ldots, x_{n}\right)$ totally omits $s(\bar{y})$ if whenever $M \models T$ and $a_{1}, \ldots, a_{n} \in M$ satisfy $\psi(\bar{x})$ then no tuple from $\left\{a_{1}, \ldots, a_{n}\right\}$ realizes $s(\bar{y})$. Let $\Sigma$ be a finite set of formulas. We simply say that $\Sigma$ totally omits $s$ if $\bigwedge \Sigma$ totally omits $s$.

Remark 5. - Let $s(\bar{x})$ be a nonisolated type. Then for every $L$-formula $\varphi(\bar{x})$ there is an $L$-formula $\varphi^{\prime}(\bar{x})$ with $T \models \varphi^{\prime}(\bar{x}) \rightarrow \varphi(\bar{x})$ such that $\varphi^{\prime}$ and $s$ are inconsistent.

- It is easy to check that for every $L$-formula $\varphi(\bar{x})$ and nonisolated type $s(\bar{y})$, there is an $L$-formula $\psi(\bar{x})$ with $T \models \psi \rightarrow \phi$ such that $\psi$ totally omits $s$.

Next lemma is easy but important for our proof of the theorem.

Lemma 6. Let $\varphi_{0}(\bar{x})$ and $\varphi_{1}(\bar{x})$ be L-formulas such that they are not essentially $L_{0}$-separable in $\bar{x}^{\prime} \subset \bar{x}$. Then $\varphi_{0}$ and $\varphi_{1}$ isolate the same complete $L_{0}$-type $p\left(\bar{x}^{\prime}\right)$.

Proof. Suppose otherwise. Then it is easy to find an $L_{0}$-formula $\xi\left(\bar{x}^{\prime}\right)$ such that both $\varphi_{0} \wedge \xi$ and $\varphi_{1} \wedge \neg \xi$ are satisfiable. Two $L$-formulas $\varphi_{0} \wedge \xi$ and $\varphi_{1} \wedge \neg \xi$ are $L_{0}$-separable in $\bar{x}^{\prime}$. Since $T \models \varphi_{0} \wedge \xi \rightarrow \varphi_{0}$ and $T \models \varphi_{1} \wedge \neg \xi \rightarrow \varphi_{1}$, this means that $\varphi_{0}$ and $\varphi_{1}$ are essentiall $L_{0}$-separable. A contradiction.

THEOREM 7. Let $R$ be a set of nonisolated complete $L_{0}$-types such that $|R|<2^{\omega}$. Let $S$ be a countalbe set of nonisolated L-types. Then there is a model $M \models T$ omitting all the members of $R \cup S$.

Proof. Suppose $Z=\left\{z_{i} \mid i<\omega\right\}$ is a fixed countable set of new variables. We denote a sequence $z_{0}, z_{1}, \ldots, z_{i-1}$ by $\bar{z}_{i}$. Enumerate $S$ as $S=\left\{s_{i}\left(\bar{x}_{i}\right): i \in \omega\right\}$. We may assume that for each $s_{n}\left(\bar{x}_{n}\right),\left|\bar{x}_{n}\right| \leq n$. Let $\left\{\theta_{i}\left(\bar{z}_{i}, z_{i}\right)\right\}$ be an enumeration of the $L$-formulas having the form $\exists x \varphi\left(\bar{z}_{i}, x\right) \rightarrow \varphi\left(\bar{z}_{i}, z_{i}\right)$.

By induction, we construct a binary tree $\left\{\Sigma_{\eta}\left(\bar{z}_{l e n(\eta)}\right) \mid \eta \in 2^{<\omega}\right\}$ of finite sets of $L$-formulas with the following properties: For every $n \in \omega$ and every $\eta \in 2^{n}$,

1. If $m<n$ then $\Sigma_{\eta \mid m} \subset \Sigma_{\eta \mid n}$;

2. $\left\{\bigwedge \Sigma_{\sigma}\left(\bar{z}_{n}\right)\right\}_{\sigma \in 2^{n}}$ is maximally separated; 
3. $\Sigma_{\eta}$ is consistent;

4. $\Sigma_{\eta}$ contains $\theta_{n}$;

5. $\Sigma_{\eta}$ totally omits each of $s_{i}(i \leq n)$.

Let $\Sigma_{\langle\rangle}=\varnothing$ and suppose $\Sigma_{\sigma}\left(\bar{z}_{n}\right)$ has been defined for every $\sigma \in 2^{n}$. Take two copies of $\Sigma_{\sigma}\left(\bar{z}_{n}\right)$ and set

$$
\Sigma_{\sigma}^{0, k}\left(\bar{z}_{n}\right)=\Sigma_{\sigma}\left(\bar{z}_{n}\right) \quad(k=0,1) .
$$

Then, by Lemma 3 , there is a set $\left\{\psi_{\sigma, k}\left(\bar{z}_{n}\right)\right\}_{\sigma \in 2^{n}, k=0,1}$ which is a maximal $L_{0}$-separation of $\left\{\bigwedge \Sigma_{\sigma}^{0, k}\left(\bar{z}_{n}\right)\right\}_{\sigma \in 2^{n}, k=0,1}$. Set

$$
\Sigma_{\sigma}^{1, k}\left(\bar{z}_{n}\right)=\Sigma_{\sigma}^{0, k}\left(\bar{z}_{n}\right) \cup\left\{\psi_{\sigma, k}\left(\bar{z}_{n}\right)\right\} .
$$

Next, for each $\sigma \in 2^{n}$, take an $L$-formula $\chi_{\sigma, k}\left(\bar{z}_{n}\right) \models \Sigma_{\sigma}^{1, k}\left(\bar{z}_{n}\right)$ such that $\chi_{\sigma, k}$ totally omits $s_{i}\left(\bar{x}_{i}\right)$ for every $i \leq n$. (Such formula exists by Remark 5.) Set

$$
\Sigma_{\sigma}^{2, k}\left(\bar{z}_{n}\right)=\Sigma_{\sigma}^{1, k}\left(\bar{z}_{n}\right) \cup\left\{\chi_{\sigma, k}\left(\bar{z}_{n}\right)\right\} .
$$

Finally set $\Sigma_{\sigma^{\wedge} k}=\Sigma_{\sigma}^{2, k}\left(\bar{z}_{n}\right) \cup\left\{\theta_{n}\left(\bar{z}_{n}, z_{n}\right)\right\}$. It is easy to check that $\left\{\Sigma_{\eta}\left(\bar{z}_{n+1}\right)\right\}_{\eta \in 2^{n+1}}$ satisfies the required conditions $1-5$ (with $n$ replaced by $n+1$ ). So we have succeeded to construct all $\Sigma_{\eta}$ 's. Now, for a path $\eta \in 2^{\omega}$, we define $\Sigma_{\eta}(Z)$ by $\Sigma_{\eta}=\bigcup_{n \in \omega} \Sigma_{\eta \mid n}$. Recall that $\theta_{n}$ has the form $\exists x \varphi\left(\bar{z}_{n}, x\right) \rightarrow \varphi\left(\bar{z}_{n}, z_{n}\right)$. So, by the condition 4 , every $M_{\eta}$ realizing $\Sigma_{\eta}(Z)$ is a model of $T$. By the condition $5, M_{\eta}$ omits all types in $S$.

Claim A. For each $p \in R,\left\{\eta \in 2^{\omega} \mid M_{\eta} \models \exists \bar{x} p(\bar{x})\right\}$ is countable.

We fix $p(\bar{x}) \in R$ and $\bar{z} \subset Z$ with $|\bar{x}|=|\bar{z}|$. Suppose $\Sigma_{\eta}(Z) \cup p(\bar{z})$ is consistent. Take any $\eta^{\prime} \neq \eta$. If $\Sigma_{\eta^{\prime}}(Z) \cup p(\bar{z})$ is also consistent, then $\Sigma_{\eta \mid n}$ and $\Sigma_{\eta^{\prime} \mid n}$ are not essentially $L_{0}$-separable in $\bar{z}$, where $n$ is chosen so that $\bar{z} \subset \bar{z}_{n}$. Hence $p$ must be isolated by a $L$-formula, by Lemma 6 . But $R$ is a set of nonisolated types, a contradiction. So, for each $p \in R$ and $\bar{z} \subset Z,\left\{\eta \in 2^{\omega} \mid \Sigma_{\eta}(Z) \cup p(z)\right.$ consistant $\}$ has at most one element. This proves the claim, since there are only countably many possible choices of $\bar{z} \subset Z$. (End of Proof of Claim)

Finally, by the claim above and the assumption that $|R|<2^{\omega}$, we can find a path $\eta \in 2^{\omega}$ such that $M_{\eta}$ omits $R$.

COROLlary 8. Suppose $\alpha<2^{\omega}$. Let $T_{0}$ be a complete L-theory and $p, q_{i} \in$ $S\left(T_{0}\right) \quad(i<\alpha)$. If for every $i<\alpha$ there is a model $M_{i}$ such that $M_{i}$ omits $q_{i}$ and $M_{i}$ realizes $p$, then there is a model $N$ such that $N$ omits all $q_{i}$ 's but $N$ realizes $p$. 


\section{Another Version of Omitting Types Theorem with Uncountably Many Types}

Recall that $L$ is a countable language and $L_{0}$ a sublanguage of $L$. In this section we show the following,

THEOREM 9. Let $T$ be a (possibly incomplete) L-theory. Let $R$ be a set of complete $L_{0}$-types with $|R|<2^{\omega}$ and $S$ a countable set of L-types. Fix an $L$-formula $\chi(x, y)$. Suppose that for any $\alpha<\omega_{1}$, there is a model $M_{\alpha}$ of $T$ containing a set $A_{\alpha} \subset M_{\alpha}$ such that

- $A_{\alpha}=\left\{a_{i}^{\alpha} \mid i \leq \alpha\right\}$,

- $M_{\alpha} \models \chi\left(a_{i}^{\alpha}, a_{j}^{\alpha}\right)$ if and only if $i<j$,

- $M_{\alpha}$ omits all the members of $R \cup S$.

Then there is a model $N \models T$ with a subset $A \subset N$ such that

- $A=\left\{a_{q} \mid q \in \mathbf{Q}\right\}$,

- $N \models \chi\left(a_{q}, a_{q^{\prime}}\right)$ if and only if $q<q^{\prime}$,

- $N$ omits all the members of $R \cup S$.

In the rest of this section, we denote $\chi(x, y)$ by $x<y$. For a tuple $\bar{a}$, the $i$ th element of $\bar{a}$ is denoted by $(\bar{a})_{i}$. We also denote the $\beta$ th element $a_{i+\beta}^{\gamma}$ from $a_{i}^{\gamma}$ in $A_{\gamma}$ by $a_{i}^{\gamma}+\beta$.

Note that if, with new constants $c_{q}(q \in \mathbf{Q}), T \cup\left\{c_{q}<c_{q^{\prime}} \mid q<q^{\prime}, q, q^{\prime} \in \mathbf{Q}\right\}$ isolates no type in $R \cup S$ then the theorem is clear by theorem 7. But, in general, $T \cup\left\{c_{q}<c_{q^{\prime}}\right\}_{q, q^{\prime}}$ may isolate some types. (Notice that $T \cup\left\{c_{q}<c_{q^{\prime}}\right\}_{q, q^{\prime}}$ may not be complete.) So we need find a theory $T^{\prime} \supset T$ that isolates no type in $R \cup S$. To construct $T^{\prime}$, we need some definitions. The following definitions are taken from the proof of Lopez-Escobar's theorem in [2].

Definition 10. 1. An $m$-sequence is a sequence of tuples of length $m$.

2. We say an ascending tuple $\bar{b} \in A_{\gamma}$ of length $m+1$ is a $k$-extension $(k \leq m)$ of an ascending tuple $\bar{a} \in A_{\gamma}$ of length $m$ if $(\bar{b})_{1}=(\bar{a})_{1}, \ldots,(\bar{b})_{k}=(\bar{a})_{k}$, $(\bar{b})_{k+2}=(\bar{a})_{k+1}, \ldots,(\bar{b})_{m+1}=(\bar{a})_{m}$.

3. Let $\Gamma$ be a subset of $\omega_{1}$. We say that the $m$-sequence $\left\{\bar{a}^{\gamma} \mid \gamma \in \Gamma\right\}$ is an unbounded $m$-sequence if

- $\Gamma$ is unbounded in $\omega_{1}$,

- $\bar{a}^{\gamma}$ is an ascending tuple of length $m$ of elements of $A_{\gamma}$, 
- for any $\beta \in \omega_{1}$ there is a $\gamma \in \Gamma$ such that $a_{\beta}^{\gamma}<\left(\bar{a}^{\gamma}\right)_{1},\left(\bar{a}^{\gamma}\right)_{1}+\beta<\left(\bar{a}^{\gamma}\right)_{2}$, $\left(\bar{a}^{\gamma}\right)_{2}+\beta<\left(\bar{a}^{\gamma}\right)_{3}, \ldots,\left(\bar{a}^{\gamma}\right)_{m}+\beta<a_{\gamma}^{\gamma}$.

4. Let $\Gamma$ be a subset of $\omega_{1}$. Let $X=\left\{\bar{a}^{\gamma} \mid \gamma \in \Gamma\right\}$ be an unbounded $m$-sequence and $Y=\left\{\bar{b}^{\gamma} \mid \gamma \in \Gamma\right\}$ an unbounded $(m+1)$-sequence. We say $Y$ is a $k$-extension of $X(0 \leq k \leq m)$ if for all $\gamma \in \Gamma, \bar{b}^{\gamma}$ is a $k$-extension of $\bar{a}^{\gamma}$.

5. We consider the unbounded 0 -sequence, the empty sequence. Every unbounded 1 -sequence is a 0 -extension of the unbounded 0 -sequence.

LEMma 11. 1. There is an unbounded 1-sequence.

2. Let $X$ be an unbounded m-sequence and $k \leq m$. Then there are an unbounded $(m+1)$-sequence $Y$ and an unbounded m-sequence $X^{\prime}$ such that $X^{\prime}$ is an unbounded m-sequence, $X^{\prime} \subset X$, and $Y$ is a k-extension of $X^{\prime}$. This condition will be denoted as $X \triangleleft_{k} Y$.

Proof. We show the second with $m=1$ and $k=0$, and the other cases are similar. Let $X=\left\{a^{\gamma}\right\}_{\gamma \in \Gamma}$ be an unbounded 1-sequence. Then for any $\beta+\beta+\beta \in \omega_{1}$ there is a $\gamma \in \Gamma$ such that $a_{\beta \cdot 3}^{\gamma}<a^{\gamma}$ (Recall $a_{\beta \cdot 3}^{\gamma}$ is the $\beta \cdot 3$-th element of $A_{\gamma}$ ). So we have a 0 -extension $\bar{b}^{\gamma}=a_{\beta}^{\gamma}+1$, $a^{\gamma}$ of $a^{\gamma}$. Collect such 0 -extension $\bar{b}^{\gamma}$ of $a^{\gamma}$ for every $\beta \in \omega$, then it is a required 2-sequence.

Take a set $C=\left\{c_{q} \mid q \in \mathbf{Q}\right\}$ of new constant symbols. To prove the theorem, it is enough to show that there is an $L$-theory $T^{\prime} \supset T \cup\left\{c_{q}<c_{q^{\prime}} \mid q<q^{\prime}\right.$ and $\left.q, q^{\prime} \in \mathbf{Q}\right\}$ such that all the members of $R$ and $S$ are nonisolated types in $T^{\prime}$, by theorem 7. We fix an enumeration $\left\{c_{q_{n}} \mid n<\omega\right\}$ of $C$. Let $\bar{c}_{n}$ be the sequence consisting $c_{q_{0}}, c_{q_{1}}, \ldots, c_{q_{n-1}}$ with the order of $\mathbf{Q}$ (e.g. if $q_{0}, q_{1}, q_{2}=0.5,-1,0$ then $\bar{c}_{3}$ is the sequence $\left.c_{-1}, c_{0}, c_{0.5}\right)$. Most ideas of the following definitions are from [8]. We adapt it to our situation.

Definition 12. Let $X=\left\{\bar{a}^{\gamma} \mid \gamma \in \Gamma\right\}$ be an unbounded $m$-sequence with $\Gamma \subset \omega_{1}$ and $\varphi(\bar{x}, \bar{c})$ an $L(\bar{c})$-formula.

1. We say $X$ is $\varphi(\bar{x}, \bar{c})$-uniform if for every $L_{0}$-formula $\xi(\bar{x})$ and $\gamma, \gamma^{\prime} \in \Gamma$, $M_{\gamma} \models \exists \bar{x}\left(\varphi\left(\bar{x}, \bar{a}^{\gamma}\right) \wedge \xi(\bar{x})\right)$ if and only if $M_{\gamma^{\prime}} \models \exists \bar{x}\left(\varphi\left(\bar{x}, \bar{a}^{\gamma^{\prime}}\right) \wedge \xi(\bar{x})\right)$

2. We say $X$ is essentially $\varphi(\bar{x}, \bar{c})$-uniform if there is an unbounded subset $\Gamma^{\prime} \subset \Gamma$ such that $X^{\prime}$ is $\varphi(\bar{x}, \bar{c})$-uniform where $X^{\prime}=\left\{\bar{a}^{\gamma} \in X \mid \gamma \in \Gamma^{\prime}\right\}$.

Lemma 13. Let $X=\left\{\bar{a}^{\gamma} \mid \gamma \in \Gamma\right\}$ be an unbounded m-sequence with $\Gamma \subset \omega_{1}$ and $\varphi(\bar{x}, \bar{c})$ an $L(\bar{c})$-formula. If $X$ is not essentially $\varphi(\bar{x}, \bar{c})$-uniform then there is an 
$L_{0}$-formula $\xi(\bar{x})$ such that $X_{\varphi \wedge \xi}$ and $X_{\varphi \wedge \neg \xi}$ are unbounded $m$-sequences where $X_{\theta(\bar{x}, \bar{c})}:=\left\{\bar{a}^{\gamma} \mid M_{\gamma} \models \exists \bar{x} \theta\left(\bar{x}, \bar{a}^{\gamma}\right)\right\}$.

Proof. Suppose that $X_{\varphi \wedge \xi}$ or $X_{\varphi \wedge \neg \xi}$ is bounded for every $L_{0}$-formula $\xi(\bar{x})$. Notice that if $X_{\varphi \wedge \xi}$ is bounded then $X_{\varphi \wedge \xi}$ is countable. So, the union $Y$ of all bounded $X_{\varphi \wedge \xi}$ 's is also countable, because $L_{0}$ is countable. Set $X^{\prime}=X \backslash Y$. Then $X^{\prime}$ is an unbounded $m$-sequence, and $X^{\prime}$ is $\varphi(\bar{x}, \bar{c})$-uniform by the definition of $X^{\prime}$. This means that $X$ is essentially $\varphi$-uniform.

Lemma 14. Let $Y$ and $Y^{\prime}$ be unbounded m-sequences. Suppose they are not essentially $\varphi_{i}(\bar{x})$-uniform for $i \leq n$. Then there is an $L_{0}$-formula $\xi_{i}(\bar{x}), X \subset Y$ and $X^{\prime} \subset Y^{\prime}$ such that $X_{\varphi_{i} \wedge \xi_{i}}=X, X_{\varphi_{i} \wedge \neg \xi_{i}}^{\prime}=X^{\prime}$ and $X, X^{\prime}$ are unbounded m-sequences, for each $i \leq n$.

Proof. We show by induction on $n$. The case $n=0$ is trivial. Let $n=k+1$. By induction hypothesis, we have $Z \subset Y$ and $Z^{\prime} \subset Y^{\prime}$ such that $Z_{\varphi_{i} \wedge \xi_{i}}=Z$, $Z_{\varphi_{i} \wedge \neg \xi_{i}}^{\prime}=Z^{\prime}$ for each $i \leq k$. Let $\xi^{0}$ be an $L_{0}$-formula dividing $Z$ into two uncountable sets $Z_{\varphi_{k+1} \wedge \xi^{0}}, Z_{\varphi_{k+1} \wedge \neg \xi^{0}}$. By shrinking $Z^{\prime}$, if necessary, we may assume $Z_{\varphi_{k+1} \wedge \neg \xi^{0}}^{\prime}=Z^{\prime}$. Then, let $\xi^{1}$ be an $L_{0}$-formula dividing $Z^{\prime}$ into two uncountable sets $Z_{\varphi_{k+1} \wedge \xi^{1}}^{\prime}, Z_{\varphi_{k+1} \wedge \neg \xi^{1}}^{\prime}$. Either $Z_{\varphi_{k+1} \wedge \xi^{0} \wedge \xi^{1}}$ or $Z_{\varphi_{k+1} \wedge \xi^{0} \wedge \neg \xi^{1}}$ is uncountable, we can take $\xi^{0} \wedge \xi^{1}$ or $\xi^{0} \wedge \neg \xi^{1}$ as $\xi_{k+1}$. Then put $X=Z_{\varphi_{k+1} \wedge \xi_{k+1}}$ and $X^{\prime}=Z_{\varphi_{k+1} \wedge \neg \xi_{k+1}}^{\prime}$.

Let $\left\{\varphi_{n}\left(\bar{x}, \bar{c}_{n}\right) \mid n<\omega\right\}$ be an enumeration of all $L(C)$-formulas. Also enumerate $S$ as $S=\left\{s_{n}\left(\bar{x}_{n}\right) \mid n<\omega\right\}$. We can assume that for every tuple $(\varphi, s) \in$ $L(C) \times S$, there is $n$ such that $(\varphi, s)=\left(\varphi_{n}, s_{n}\right)$. So, each member of $L(C), S$ appears infinitely many times in the enumerations. By induction, we construct a binary tree $\left\{T^{\sigma}\left(\bar{c}_{l e n(\sigma)}\right) \mid \sigma \in 2^{<\omega}\right\}$ of sets of $L(C)$-formulas and unbounded len $(\sigma)$ sequence $X^{\sigma}=\left\{\bar{a}_{\gamma} \mid \gamma \in \Gamma_{\sigma}\right\}$ with the following properties: For every $\sigma, \sigma^{\prime} \in 2^{<\omega}$ and $n \leq \operatorname{len}(\sigma)$,

1. $T^{\sigma}\left(\bar{c}_{l e n(\sigma)}\right) \cup\left\{c_{q}<c_{q^{\prime}} \mid c_{q}, c_{q^{\prime}} \in \bar{c}_{l e n(\sigma)}\right.$ and $\left.q<q^{\prime}\right\}$ is consistent,

2. $\sigma \subset \sigma^{\prime}$ then $T^{\sigma} \subset T^{\sigma^{\prime}}$,

3. $M_{\gamma}, \bar{a}_{\gamma} \models T^{\sigma}\left(\bar{c}_{l e n(\sigma)}\right)$ for uncountably many $\gamma \in \Gamma_{\sigma}$,

4. $T^{\sigma}$ contains $\exists \bar{x} \varphi_{\operatorname{len}(\sigma)}$ or $\neg \exists \bar{x} \varphi_{\operatorname{len}(\sigma)}$,

5. $T^{\sigma}$ contains $\exists \bar{x}\left(\varphi_{\text {len }(\sigma)}\left(\bar{x}, \bar{c}_{l e n(\sigma)}\right) \wedge \neg \psi(\bar{x})\right)$ for some $\psi \in s_{l e n(\sigma)}$,

6. if $X^{\sigma}$ is essentially $\varphi_{n}$-uniform then it is $\varphi_{n}$-uniform,

7. if $X^{\sigma}$ is not essentially $\varphi_{n}$-uniform then $\exists \bar{x}\left(\xi(\bar{x}) \wedge \varphi_{n}\left(\bar{x}, \bar{c}_{l e n(\sigma)}\right)\right) \in T^{\sigma^{\wedge} 0}$ and $\exists \bar{x}\left(\neg \xi(\bar{x}) \wedge \varphi_{n}\left(\bar{x}, \bar{c}_{l e n(\sigma)}\right)\right) \in T^{\sigma^{\wedge} 1}$ for some $\xi \in L_{0}$. 
Let $T^{\langle>}=\varnothing, X^{<>}$the unbounded 0 -sequence and suppose $T^{\sigma}\left(\bar{c}_{n}\right)$ and $X^{\sigma}$ are defined for every $\sigma \in 2^{n}$. Suppose $\bar{c}_{n+1}$ is a $k$-extension of $\bar{c}_{n}$. Take an unbounded $(n+1)$-sequence $Y^{\sigma} \triangleright_{k} X^{\sigma}$ (Lemma 11). If $Y_{\varphi_{n+1}}^{\sigma}$ is uncountable, set

$$
Y^{\sigma, 0}=Y_{\varphi_{n+1}}^{\sigma}
$$

and

$$
T^{\sigma, 0}=T^{\sigma} \cup\left\{\exists \bar{x} \varphi_{n+1}\left(\bar{x}, \bar{c}_{n+1}\right)\right\}
$$

otherwise

$$
\begin{gathered}
Y^{\sigma, 0}=Y_{\neg \varphi_{n+1}}^{\sigma}, \\
T^{\sigma, 0}=T^{\sigma} \cup\left\{\neg \exists \bar{x} \varphi_{n+1}\left(\bar{x}, \bar{c}_{n+1}\right)\right\} .
\end{gathered}
$$

Recall that $s_{n+1}\left(\bar{x}_{n+1}\right)$ is countable and $M_{\alpha}$ omits $s_{n+1}\left(\bar{x}_{n+1}\right)$ for every $\alpha$. Hence, we can find $\psi\left(\bar{x}_{n+1}\right) \in s_{n+1}$ such that $Y_{\varphi_{n+1} \wedge \neg \psi}^{\sigma, 0}$ is uncountable. Set

$$
\begin{gathered}
Y^{\sigma, 1}=Y_{\varphi_{n+1} \wedge \neg \psi}^{\sigma, 0}, \\
T^{\sigma, 1}=T^{\sigma, 0} \cup\left\{\exists \bar{x}\left(\varphi_{n+1}\left(\bar{x}, \bar{c}_{n+1}\right) \wedge \psi(\bar{x})\right)\right\} .
\end{gathered}
$$

Then, if $Y^{\sigma, 1}$ is essentially $\varphi_{n+1}$-uniform, by shrinking it, we may assume $Y^{\sigma, 1}$ is $\varphi_{n+1}$-uniform.

Finally, we consider the $\varphi_{j}$-uniformity of $Y^{\sigma, 1}(j \leq N+1)$. If $Y^{\sigma, 1}$ is $\varphi_{j}$ uniform for every $j \leq n+1$ then set $X^{\sigma^{\wedge} 0}=X^{\sigma^{\wedge} 1}=Y^{\sigma, 1}$ and $T^{\sigma^{\wedge} 0}=T^{\sigma^{\wedge} 1}=T^{\sigma, 1}$. Otherwise, assume $Y^{\sigma, 1}$ is not essentially $\varphi_{j}$-uniform for some $j$. Then, take an unbounded $(n+1)$-sequence $X^{\sigma^{\wedge} i} \subset Y^{\sigma, 1}(i=0,1)$ such that for all $j \leq n$, if $Y^{\sigma, 1}$ is not essentially $\varphi_{j}$-uniform, then $X_{\varphi_{j} \wedge \xi_{j}}^{\sigma^{\wedge}}=X^{\sigma^{\wedge} 0}$ and $X_{\varphi \wedge \neg \xi_{j}}^{\sigma^{\wedge}}=X^{\sigma^{\wedge} 1}$ for some $\xi_{j}(\bar{x}) \in L_{0}$ (See lemma 14). We set

$$
\begin{gathered}
T^{\sigma^{\wedge} 0}=T^{\sigma, 1} \cup\left\{\exists \bar{x}\left(\varphi_{j}\left(\bar{x}, \bar{c}_{j}\right) \wedge \xi_{j}(\bar{x})\right\}_{j},\right. \\
T^{\sigma^{\wedge} 1}=T^{\sigma, 1} \cup\left\{\exists \bar{x}\left(\varphi_{j}\left(\bar{x}, \bar{c}_{j}\right) \wedge \neg \xi_{j}(\bar{x})\right\}_{j} .\right.
\end{gathered}
$$

It is easy to check that they satisfy the required conditions. At the end of this inductive construction, we have $2^{\omega}$ complete $L(C)$-theories $T^{\eta}\left(\eta \in 2^{\omega}\right)$. By condition 5. and the way of enumerations of $L(C)$ and $S$, every member of $S$ is not isolated in $T^{\eta}$.

Claim A. The set $\left\{\eta \in 2^{\omega} \mid p\right.$ is isolated in $\left.T^{\eta}\right\}$ is countable for every $p \in R$.

Suppose $p(\bar{x})$ is isolated by an $L(C)$-formula $\varphi_{n}\left(\bar{x}, \bar{c}_{n}\right)$ in $T^{\eta}$ and $T^{\eta^{\prime}}$. If $X^{\eta \mid n}=\left\{\bar{a}_{\gamma} \mid \gamma \in \Gamma_{\eta \mid n}\right\}$ is $\varphi_{n}$-uniform then $M_{\gamma}\left(\gamma \in \Gamma_{\eta \mid n}\right)$ realizes $p(\bar{x})$. So, $X^{\eta \mid n}$ is 
not essentially $\varphi_{n}$-uniform. We can assume that $\eta\left|n \neq \eta^{\prime}\right| n$, because, by condition 7., if $\eta\left|n=\eta^{\prime}\right| n, \varphi_{n}$ cannot isolates same complete $L_{0}$-type in $T^{\eta}, T^{\eta^{\prime}}$. Therefore, $\left\{\eta \in 2^{\omega} \mid p\right.$ is isolated in $\left.T^{\eta}\right\}$ is countable. (End of proof of claim)

Hence, there is an $\eta \in 2^{\omega}$ such that every member of $R$ is nonisolated in $T^{\eta}$. By theorem 7, we have a required model. (End of proof of theorem 9)

\section{Omitting Types Theorem with Nonelementary Classes}

In this section, we look at the definitions of some nonelementary classes. Then we have Shelah's omitting types theorem for such classes.

Definition 15. Let $\mathscr{K}$ be a class of $L$-structures. We say $\mathscr{K}$ is an $E C\left(\aleph_{0}, \aleph_{0}\right)$-class if

- $L$ is countable,

- there is a countable set $S$ of types and an $L$-theory $T$ such that $M \in \mathscr{K}$ if and only if $M \models T$, and $M$ omits all the members of $S$.

$\mathscr{K}$ is denoted by $E C(T, S)$.

More general definitions and properties of $E C(\kappa, \lambda)$ can be found in [1]. It is well known that every $E C\left(\aleph_{0}, \aleph_{0}\right)$-class can be translated to a class defined by an $L_{\omega_{1}, \omega}$-sentence, and vice versa (see [7], for example). Next, we introduce a $P C_{\delta}$-class. This is defined by Keisler in [2] with $L_{\omega_{1}, \omega}$. The following definition of a $P C_{\delta}$-class is given without $L_{\omega_{1}, \omega}$. Note that Shelah and Baldwin use other notations, e.g., $P C\left(\aleph_{0}, \aleph_{0}\right), P C \Gamma\left(\aleph_{0}, \aleph_{0}\right)$ (see [1]).

Definition 16. Let $\mathscr{K}$ be a class of $L$-structures. We say that $\mathscr{K}$ is a $P C_{\delta}$-class if there is a countable language $L^{\prime} \supset L$ and a class of $L^{\prime}$-structures $\mathscr{K}^{\prime}$ such that

- $K^{\prime}$ is an $E C\left(\aleph_{0}, \aleph_{0}\right)$-class, and

- $M^{\prime} \mid L \in \mathscr{K}$ if and only if $M^{\prime} \in \mathscr{K}^{\prime}$ for every $L^{\prime}$-structure $M^{\prime}$.

To generalize the omitting types theorem for a $P C_{\delta}$-class, we need definitions of types and isolated types.

Definition 17. Let $\mathscr{K}$ be a class of $L$-structures. A type $\Sigma(\bar{x})$ in $\mathscr{K}$ is a set of $L$-formulas with free variables $\bar{x}$ such that there is a structure $M \in \mathscr{K}$ having a realization of $\Sigma(\bar{x})$. 
Definition 18. Let $\mathscr{K}$ be either $E C(T, S)$ or the $P C_{\delta}$-class obtained from $E C(T, S)$ by restricting the language. An isolated type in $\mathscr{K}$ is a type in $\mathscr{K}$ which is isolated in $T$ in the usual sense.

The above definitions and theorem 7 immediately give Shelah's omitting types theorem for $P C_{\delta}$-classes.

THEOREM 19. Let $\mathscr{K}$ be a $P C_{\delta}$-class. Let $R$ be a set of nonisolated complete types in $\mathscr{K}$ such that $|R|<2^{\omega}$. Then there is a model $M \models T$ omitting all the members of $R$.

We also have Lopez-Escobar's theorem (with uncountably many types) for $P C_{\delta}$-classes.

THEOREM 20. Let $\mathscr{K}$ be a $P C_{\delta}$-class with a countable language L having a binary relation $<$. Let $R$ be a set of complete L-types such that $|R|<2^{\omega}$. Suppose that for any $\alpha<\omega_{1}$, there is a model $M_{\alpha} \in \mathscr{K}$ omitting $R$ and with the order type $\alpha$. Then there is a model $N \in \mathscr{K}$ omitting $R$ and with the order type $\mathbf{Q}$.

\section{References}

[1] Baldwin, J. T., Categoricity, Amer Mathematical Society, 2009.

[2] Keisler, H. J., Model theory for Infinitatry Logic. North-Holland, 1971.

[3] Lopez-Escobar, E. G. K., "On definable well-orderings," Fundamenta Mathmatica, vol. 59 (1966), pp. 13-21, 299-300.

[4] Newelski, L., "Omitting types and the real line," The Journal of Symbolic Logic, vol. 52 (1987), pp. $1020-1026$.

[5] Shelah, S., "Models with second order properties. III. Omitting types for $L(Q)$ " Archiv fur Math Logik und Grundlagenforschung, vol. 21 (1981), pp. 1-11 GgSh:83.

[6] Shelah, S., Classification Theory and the Number of Nonisomorphic Models, North-Holland, 1991. second edition.

[7] Takeuchi, K., "Completeness and The Number of Types for Infinitary Logic," RIMS Kokyuroku, vol. 1718 (2010) Reserch Institute for Mathmatical Sciences, Kyoto University, pp. $75-80$.

[ 8 ] Tsuboi, A., "Models omitting Given Complete Types," Notre Dame Journal of Formal Logic, vol. 49 (2008), number 4, pp. 393-399.

Graduate School of Pure and Applied Sciences University of Tsukuba

E-mail: kota@math.tsukuba.ac.jp 